undermined by those who should be its defenders. Underlying the attacks on freedom of thought and of the Press is the dangerous proposition that freedom is undesirable and that intellectual honesty is a form of anti-social selfishness. The enemies of intellectual liberty try to present their case as a plea for discipline versus individualism, leaving in the background that the issue is truth versus untruth. Totalitarianism, whether political or religious, exerts its greatest pressure on the intellectual at the point where literature and politics cross. The exact sciences are not, so far, menaced to the same extent, yet some scientific workers seem to think that the destruction of liberty is of no importance so long as their own line of work is unaffected. Even totalitarian States tolerate the scientific worker for the moment because his work is recognized by the rulers as necessary, if only to prepare for war. But when the totalitarian State will be well established, this may not be so. If, therefore, the man of science would guard the integrity of science, he should develop some kind of solidarity with his literary colleagues. In Great Britain, broadly speaking, there is liberty ; but there is the sinister suggestion that the conscious enemies of liberty are those to whom liberty ought to mean much. 516

\section{R.C.A. Review}

AlL those concernga yith radio research and development will weledn the reappearance, after a four-yequ interval, of th R.C.A. Review, a technical journal beorfling Argress in radio and electronics resteh and engmeering as described by scientific workers, endreers and executives of the Radio Corporation of America. The March 1946 issue forms the first number of volume 7 ; and in an introduction thereto General David Sarnoff, president of the Radio Corporation of America, explains that the new R.C.A. Review is written by men of science and engineers not only to relate their past achievements but also to reflect the thoughts of those whose pioneering in research, development and engineering are projecting the present into the future. The eight papers in the present issue cover a variety of subjects relating to television, navigation and radio telegraphic signalling by change of frequency in contrast with on-off keying. The experimental results obtained in the development of omni-directional radio beacons for aerial navigation are described in a paper by D. G. C. Luck, of which the first two parts were published in 1941 and 1942. Another paper, by I. F. Byrnes, discusses the possibilities of a shipboard radar installation as an aid to navigation for the mercantile marine. The development of the image orthicon tube for an extremely sensitive television camera is described by R. D. Kell and G. C. Szikali of the Research Dept., R.C.A. Laboratories; while some of the results obtainable by the use of this camera for field television operation are dealt with by R. E. Shelby and H. P. Lee, of the National Broadcasting Company, Inc. Short biographical notes with photographs of all the contributors form an inffesting appendix to this publication.

New 3 its for thy, Measurement of Radioactivity

Two new unj 'the 'rutherford' and the 'roentgenper-hour atpotie metre' for the measurement of radioagtifify, have recently been recommended by the National Bureau of Standards, at the suggestion of he Committee on Radioactivity of the National Research Council. The Radiology Congress in
Brussels in 1910 defined. the curie as "the amount of radon in equilibrium with one gram of radium". Therefore, strictly speaking, the curie can only be used to represent the disintegration-rate of radium or its equilibrium products. As is pointed out by E. U. Condon and L. F. Curtiss (Rev. Sci. Instr., 17, 249 ; June 1946. Also Phys. Rev., 69, 672; June 1946), it has, however, become the custom to use the curie, quite erroneously, as the unit of strength of all radioactive sources. The disintegration. rate, which correctly specifies the strength of a radioactive source, is a pure number, and is determined by the decay constant and the number of atoms of the radioactive isotope in the source. All that is required, therefore, to establish a proper unit, is to choose a suitable number, preferably a multiple of ten. The number $10^{6}$, with the name 'rutherford', abbreviated to 'rd.', is recommended. No confusion can arise when dealing with the radium family, as the curie and rutherford are sufficiently different in magnitude. Apart from that of definition, the rutherford has other advantages over the curie. The rutherford is a definite unit, whereas the curie is uncertain to at least 4 per cent, and also, the new unit does away with the necessity for measuring radio-isotopes in terms of a standard. For the intensity of gamma-ray sources, it is recommended that a roentgen-per-hour at one metre be used in place of the curie. The abbreviation, 'r.h.m.', pronounced 'rum', is suggested. A gamma-ray source of one r.h.m. has a gamma-ray strength of the same order of magnitude as that of one curie of radium.

A Thermal Eyepiece formene Telescope $\$ / 6$ AN article with tots by H. P. Wilkins, an assiduous obserefof the moon, who recently produced a 300-in Jund map appears in Sky and Telescope of May. Wr. "Wilkins has made a 'thermal eyepiece' (i) fis unly when it is inserted in the focus of his $12 \frac{1}{2}$-in. Notonian reflector. The instrument consists of a very small light couple suspended between the poles of a permanent magnet, one junction of the couple being at the focus of a positive eyepiece of orthoscopic or monocentric type. It is necessary to suspend the couple freely, and hence in the case of an equatorial mounting the tube or eyepiece should rotate. The apparatus is enclosed in a brass case which fits into the sliding eyepiece tube like an ordinary eyepiece. The couple is made from strips of copper and constantan, and the suspension is a very fine quartz thread. Full directions are given about the use of the instrument, the principle of which depends upon the fact that the thermo-electric current produced by the heat of the moon causes the loop to turn in the field of the permanent magnet, and it is possible to judge the relative intensities of radiation from various portions of the lunar surface. The apparatus can be shielded from the heat of the body by interposing a non-conducting sheet of wood or asbestos. Interesting results have been obtained during lunar eclipses, the turning moment of the couple diminishing before the encroachment of the umbra and being reduced to zero during totality. During penumbral eclipses, when the sun, as seen from the moon, appears partially eclipsed by the earth, a diminution of the heat from the moon is noticed. It seems possible for most amateurs with a small mechanical equipment to construct one of these instruments, though many details must be attended to; these are dealt with very fully in the article. 